\title{
The Research Fronts and Hotspots on Nanotechnology Based on Journal of Vacuum Science \& Technology
}

\author{
Wang Yi ${ }^{1}$, Mao Di ${ }^{2}$ \\ ${ }^{1}$ The Center for Information and Strategic, Suzhou Institute of Nano-Tech and Nano-Bionics, Chinese Academy \\ of Sciences, Suzhou, China \\ ${ }^{2}$ Department of Subject Information Service, Wuhan Branch of the National Science Library, Chinese Academy \\ of Sciences, Wuhan, China \\ Email: ywang2008@sinano.ac.cn, maod@whlib.ac.cn
}

Received 11 February 2016; accepted 26 February 2016; published 1 March 2016

\begin{abstract}
Journal of Vacuum Science \& Technology (JVST) serial journals are the official publications of American Vacuum Society. We search the nanotechnology-related references on JVST and obtain visual maps through a visual analysis tool, CiteSpace. The visual maps can help us understand the distribution of research institutions and cooperation, high-impact authors, core literatures, research hotspots and fronts of nanotechnology in the field of vacuum.
\end{abstract}

\section{Keywords}

Nanotechnology, Vacuum, Citespace, Visual Maps, Research Fronts, Research Hotspots

\section{Introduction}

CiteSpace is used to detect and analyze the emerging trends in the research fronts of subjects, and the relationship of their research fronts, intellectual bases and other research fronts. It can be more intuitive to recognize the evolving paths of subject fronts and classic basal literatures in subject areas by employing the visualized literature information. It is mainly equipped with the following visual features: co-cited articles analysis, co-occurring terms analysis, institution cooperation analysis, author co-citation analysis, authors cooperation analysis and so on. In addition, one of important features of CiteSpace is detection of burst term which relies on the level of frequency and trend of the words, owning high frequency change rates detected from a lot of phrases by examining the time distribution of frequency, to determine the fronts of the evolution of a field. We analyze the nanotechnology-related references on JVST by using CiteSpace to obtain visual maps. The visual maps can help us understand the distribution of research institutions and cooperation, high-impact authors, core literatures, research hotspots and fronts of nanotechnology in the field of vacuum. 


\section{Data Source AND Visual Tool}

\subsection{Data Source}

This article uses the Science Citation Index Expanded database of online SCI(Thomson. ISI), Web of Science, as a data source. We search 4040 records in total by choosing advanced search as the search method and query is (so = Journal of Vacuum Science \& Technology*) and (ts = nano* or ts = graphene). Data Download mode is set to "Record and contain the whole cited references," and the data download time ends in September 26, 2013.

\subsection{Visual Tool}

Citation analysis visualization is an important branch of information visualization, which deals with a flood of citation data at first, and then makes it easier to observe, explore and understand the information to find the hidden data patterns and models by using information visualization technology. Citation analysis visualization tools as used herein are based on CiteSpace 3.7.R5 of JAVA platform. The CiteSpace 3.7.R5 is developed by By Chen Chaomei, a doctor in the College of Information Science and Technology, Drexel University in Philadelphia, USA. He is the international authority in the field of information visualization and devoted to study the information visualization research methods and related algorithms for many years.

CiteSpace is used to detect and analyze the emerging trends in the research fronts of subjects, and the relationship of their research fronts, intellectual bases and other research fronts. It can be more intuitive to recognize the evolving paths of subject fronts and classic basal literatures in subject areas by employing the visualized literature information. It mainly is equipped with the following visual features: co-cited articles analysis, co-occurring terms analysis, institution cooperation analysis, author co-citation analysis, authors cooperation analysis and so on. In addition, one of important features of CiteSpace is detection of burst term which relies on the level of frequency and trend of the words, owning high frequency change rates detected from a lot of phrases by examining the time distribution of frequency, to determine the fronts of the evolution of a field [1]-[3].

\section{Result Analysis}

\subsection{The Analysis for the Distribution and Cooperation of Research Institutions}

Citespace shows immediately the amounts and issued time of articles in every country (region) by the size and color of "Ring" [4]. In the interface setting of CiteSpace software, we still choose 1985 to 2013 as the time interval, 3 years as the time span, the title, abstract, author keywords and keywords plus in the article as subject terms, authors and cited authors as node type in the network configuration. Criterion selects "Top N per slice" and top 50 mostly cited or occurred items from each slice, and other options remain unchanged. As a result, the analysis map of research countries can be obtained by operate this software. The map contains 74 nodes and 39 connections.

As shown in Figure 1, every node represents a country. The size of node reflects the amount of articles, and the width of the color of "Ring" means the article amount in the corresponding time period. The connections indicate the cooperation between countries, and the colors of them mark the first cooperation time. Select of 100, which issued countries, there are a total of eight countries issuing more than 100 articles by selecting 100 as the display threshold value. Specific statistic data can be seen in Table 1.

\subsection{The Analysis for the Distribution and Cooperation of Research Institutions}

Use a zero before decimal points: “ 0.25 ”, not “.25”. Use " $\mathrm{cm}^{3}$ ”, not "cc". As can be seen from Figure 2, the main cooperation of these institutions is divided into three relatively concentrated areas. There are 4040 articles which generate 663 nodes and 1436 connection, so most institutions cooperated with each other for the first time during 1990-2000 year. The quite important nodes are MIT, Meiji University, University of Glasgow, University of Pittsburgh and The Hong Kong Polytechnic University. In Table 2, these institutions' rankings are determined by the influence of issued statistics (issued number more than 60).

\subsection{The Analysis for Author Co-Citation}

White, the doctor of Drake University in USA, believes that the higher frequency of author's co-citation, the higher relevance in the academic of authors [5] [6]. In the interface setting of CiteSpace software, we still 


\section{GERMANY}

\section{FRANCE}

\section{USA ${ }^{E N G L A N D P L E S ~ R ~ C H I N A ~}$}

TAIWANAPAN

Figure 1. The distribution and cooperation of research institutions.

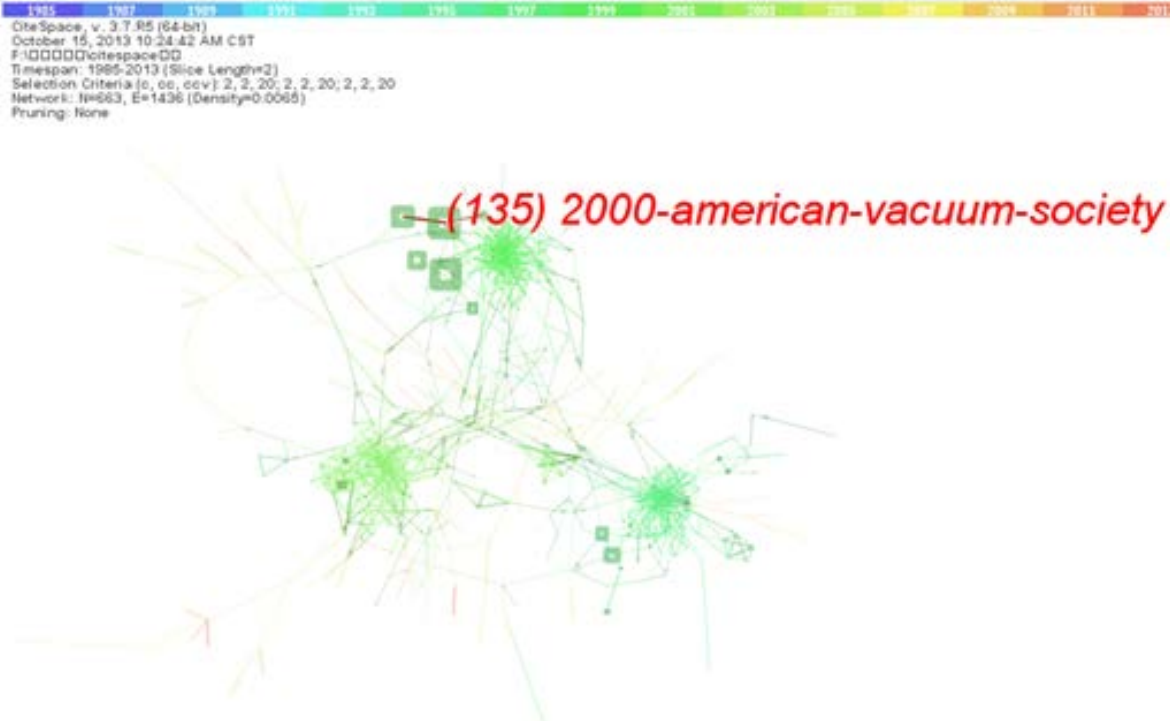

Figure 2. The distribution and cooperation of research institutions.

Table 1. Research institutions and the number of issued articles.

\begin{tabular}{cccccccc}
\hline Organization & MIT & IBM CORP & OSAKA UNIV & UNIV ILLINOIS & USN & UNIV WISCONSIN & CHINESE ACAD SCI \\
\hline No. Articles & 96 & 83 & 68 & 66 & 64 & 63 & 62 \\
\hline
\end{tabular}

choose 1985 to 2013 as the time interval, 3 years as the time span, the title, abstract, author keywords and keywords plus in the article as subject terms, authors and cited authors as node type in the network configuration. Criterion selects “Top N per slice” and top 50 mostly cited or occurred items from each slice, and other options remain unchanged. As a result, the analysis map of author co-citation can be obtained by operate this software. The map contains 332 nodes and 887 connections. Namely, these 4040 articles have 332 authors in total whose citations are higher than the threshold value. The co-citation relationships are shown in Figure 3. Table 2 exhibits the data statistics of articles and citations of the authors ranking top 10. 


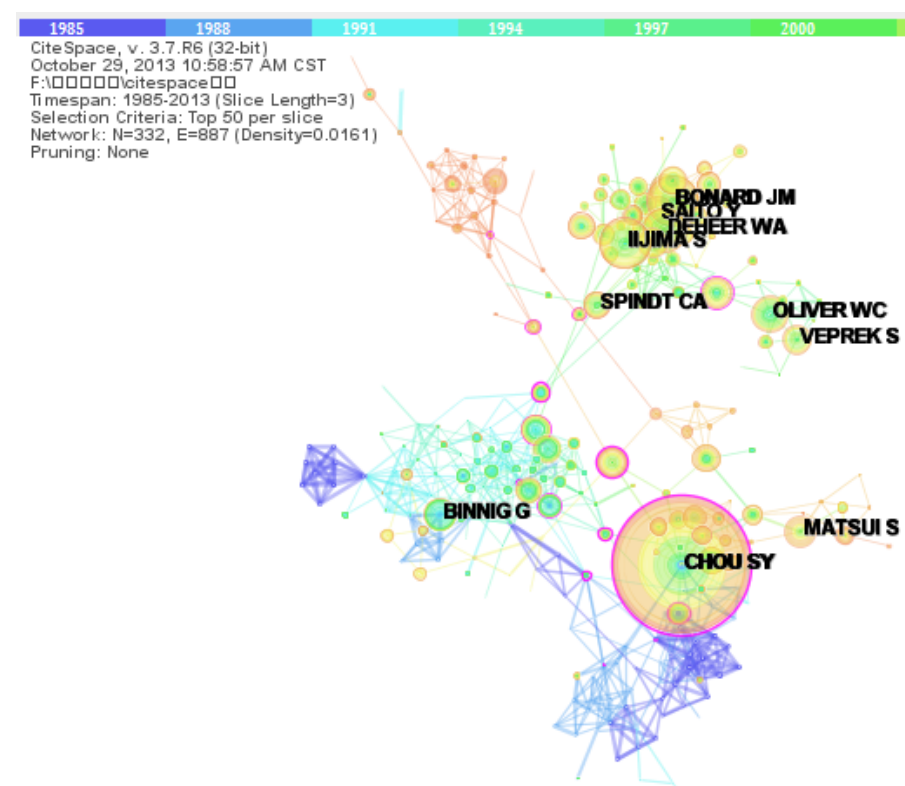

Figure 3. The analysis for author co-citation.

Table 2. The data statistics of articles and citations of the authors ranking top 10 .

\begin{tabular}{ccccc}
\hline Rank & No. Articles & Centrality & The year of the first paper & Author \\
\hline 1 & 277 & 0.35 & 1986 & CHOU SY \\
2 & 112 & 0 & 2001 & BONARD JM \\
3 & 110 & 0.08 & 1991 & IIJIMA S \\
4 & 101 & 0.03 & 1995 & DEHEER WA \\
5 & 90 & 0.03 & 1998 & SAITO Y \\
6 & 83 & 0.02 & 1986 & BINNIG G \\
7 & 82 & 0.03 & 1992 & OLIVER WC \\
8 & 79 & 0.03 & 1984 & MATSUI S \\
9 & 78 & 0.07 & 1976 & SPINDT CA \\
\hline
\end{tabular}

Firstly, we discern that the most influent author is CHOU SY in this field due to his articles higher than the threshold value are 277 , and centrality is 0.35 . Therefore, he should be regarded as the most authoritative expert in this field, and his researches have profound influences. The spatial distribution in Table 2 reflects the authors' active time, which is earlier in the bottom. CHOU SY, BINNIG G and MATSUI S are prior researchers because of their earlier active time. Then it can be seen from the color of cited annual rings, the time of the citation of BINNIG G's and MATSUI S's researches is mainly before and after 2000, respectively. The time of the main publications and citations of VEPREK S, OLIVER WC, SPINDT CA, IIJIMA S, DEHEER WA, SAITO Y and BONARD JM's researches are gradually rearward, so we can acquire the research frontal intellectual to some extent through analyzing them. Moreover, as the relevance of their researches is rather great, we are capable of speculating the latest study hotspots by analyzing their research overlaps.

\subsection{The Analysis for Intellectual Base}

The settings of CiteSpace choose 1985 to 2013 as the time interval, 3 years as the time span, the title, abstract, author keywords and keywords plus in the article as subject terms, cited reference as node type in the network 
configuration. Criterion selects “Top N per slice”, top 50 most cited or occurred items from each slice, and the key pathfinder algorithm. Other options remain unchanged. In the scientific knowledge map of the co-cited articles creating by CiteSpace, 365 citations and 630 connections between them are selected. As shown in Figure 4 (in the figure, every node represents one reference, the thickness of a dot is in proportion to the number of citations in the corresponding year): in Figure 4, the important nodes reflex the intellectual base in the field of nanotechnology, which reveal 8 core references in all. The author, title, Published year, citation and co-cited Freq in the CiteSpace algorithm of articles are summarized in Table 3.

It can be found that 4 of 8 core node references come from Chou SY by analyzing Figure 4 and Table 3. Moreover, the relevance of these articles is relatively great, and their cited time is mainly after 2000, especially recent years. From analysis of references in the key nodes, we also find that the major researches are concentrated in the nanometer lithography, SWNT technology and other material property orientations.

Furthermore, we make a map of the annual change distribution of all references for them and co-cited relationships. That is a map depending on the time zone in Figure 5. There are 534 nodes and 1004 connections, and the pivotal references are similar to them in Figure 4. The connections and color are to better reflect the referenced relationship between literatures.

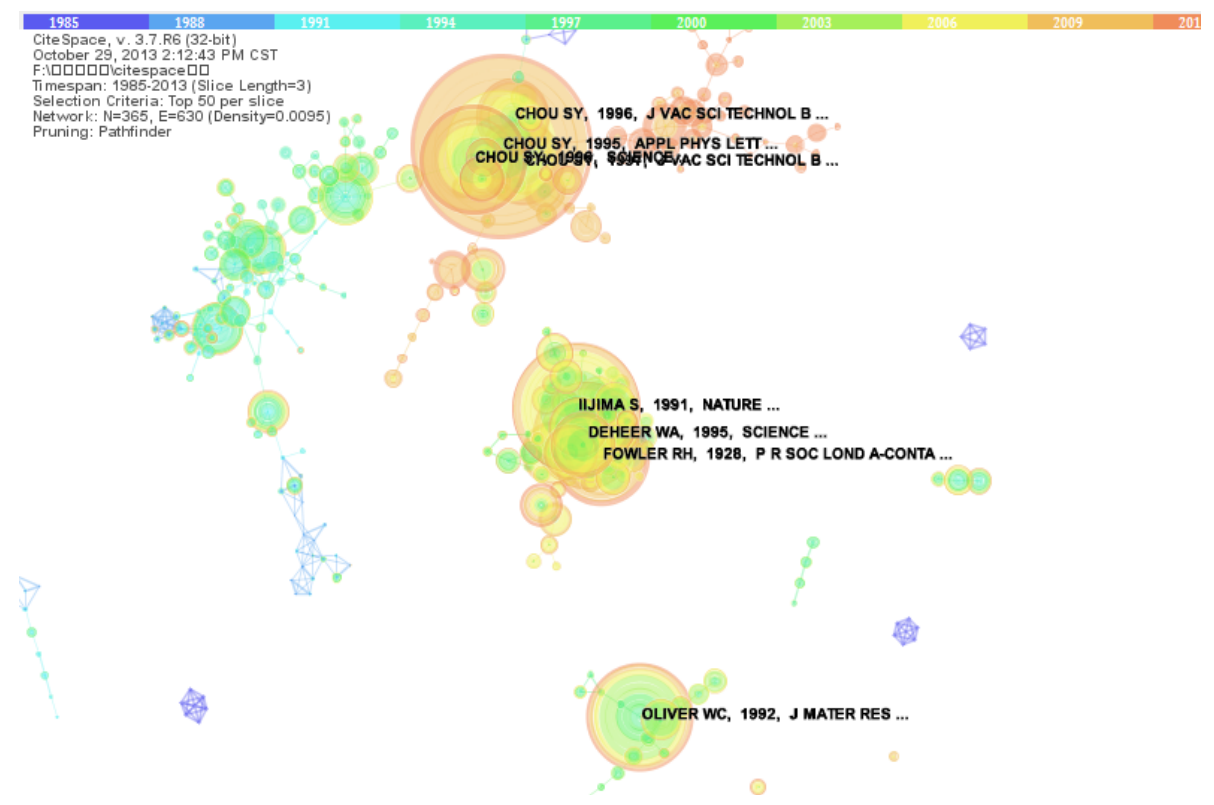

Figure 4. Co-citation network.

Table 3. The information of the core references.

\begin{tabular}{ccccc}
\hline First author & Year & Title & Cited times & Co-citations \\
\hline Chou, SY & 1995 & IMPRINT OF SUB-25 NM VIAS AND TRENCHES IN POLYMERS & 1488 & 131 \\
IIJIMA S & 1991 & HELICAL MICROTUBULES OF GRAPHITIC CARBON & 21226 & 95 \\
Chou, SY & 1996 & Imprint lithography with 25-nanometer resolution & 1436 & 80 \\
DEHEER WA & 1995 & A CARBON NANOTUBE FIELD-EMISSION ELECTRON SOURCE & 2318 & 78 \\
& & AN IMPROVED TECHNIQUE FOR DETERMINING HARDNESS & 8371 & 78 \\
OLIVER WC & 1992 & AND ELASTIC-MODULUS USING LOAD AND DISPLACEMENT & & 73 \\
FOWLER RH & 1928 & SENSING INDENTATION EXPERIMENTS & 2956 & 61 \\
Chou, SY & 1997 & Electron emission in intense electric fields & 673 & 518 \\
Chou, SY & 1996 & Sub-10 nm imprint lithography and applications & 57 \\
\hline
\end{tabular}




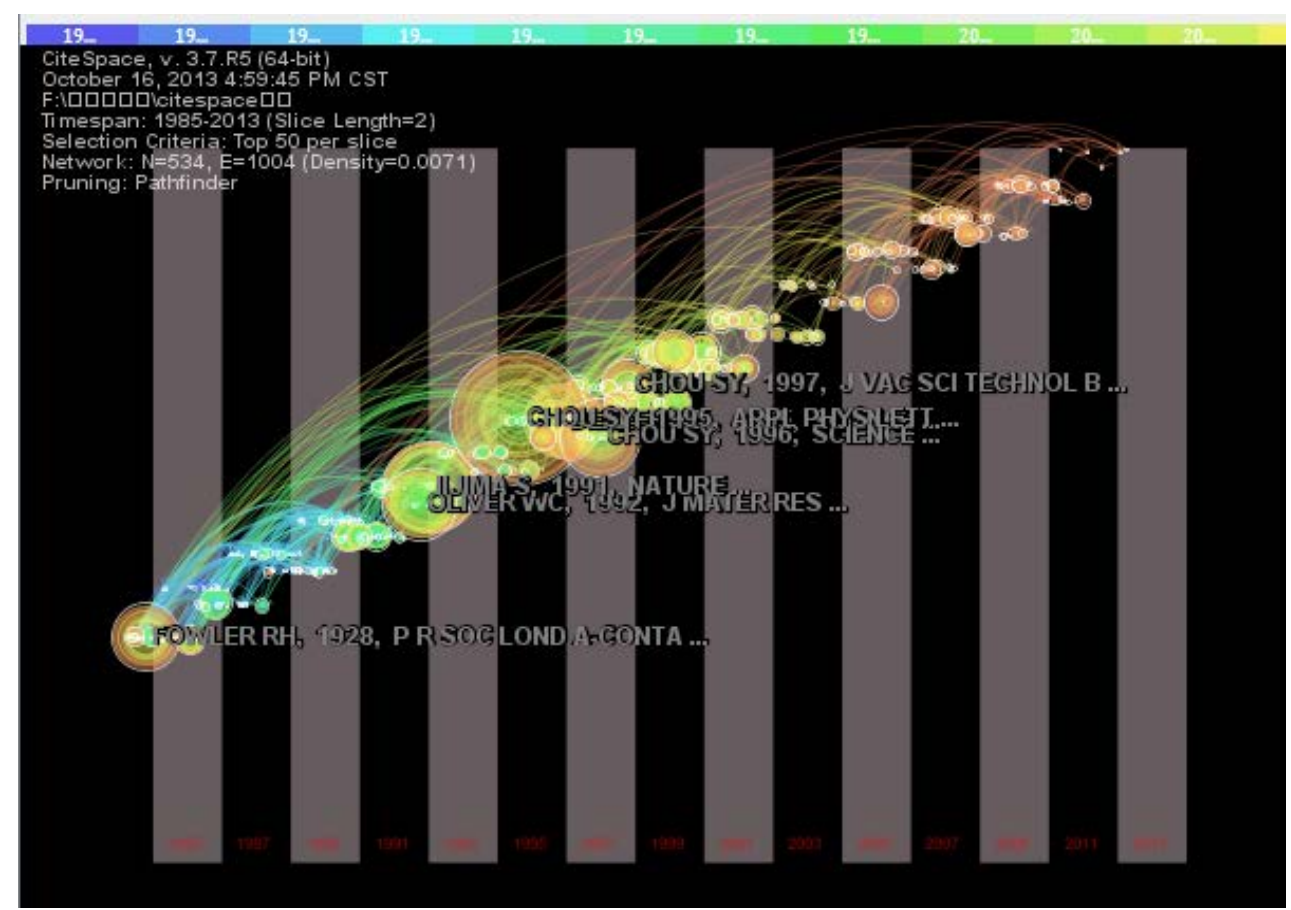

Figure 5. The map of reference co-citations depending on the time zone.

\subsection{The Analysis for Hotspots}

Co-occurring terms analysis mirrors the hotspots in this field. The node type of the network configuration selects keywords pathfinder algorithm, and other options remain unchanged. We can obtain the hotspots from clustering analysis of the map of co-occurring terms. There are 174 nodes and 255 connections in Figure 6.

As can be seen in Table 4, the co-cited frequencies of keywords in the top 10 are extremely high, which reflect approximately the research fronts and hotspots. These words are still active keywords to the present from the annual color.

It can get 19 clusters by selecting "Find optimal clusters" and then can get 15 clusters by selecting "Label clusters with title terms" in the keywords map in Figure 7.

\subsection{The Analysis for Research Fronts and Trend}

The concept of research fronts is proposed firstly by Price, who thinks research fronts can descript the dynamic nature of research fields. Garfield defines research fronts as the set of the core highly cited papers and citing papers, and believes that the name of research fronts can be extracted from the words or phrases which have the highest frequency of occurrence in the these paper titles. Dr. Chen Chaomei defined research fronts as a set of emergent dynamic concepts and potential research questions, and the intellectual base of research fronts was quotations and Co-citation Tracks. Citespace determines the net knowledge map of research fronts by the mutant terms abstracted from the titles, abstracts, index terms and record identifiers of references. Specific methods are selecting Cited Reference as the net node, "Top N per slice" and top 15 most cited or occurred items from each slice as threshold willing, and key pathfinder algorithm. After operating it, we receive 19 clusters by choosing "Find optimal clusters" and the cluster name by selecting "Label clusters with title terms". The generated knowledge map is shown in Figure 8.

There are 143 nodes, 186 links and 19 clusters. Every cluster has a different area. The larger area cluster contains, the more bibliography entry it has, and this cluster is the more main research directions. In Figure 8 and Table 5, it shows that the research fronts and trends major in scanning tunneling microscopy, bidirectional atomic force microscope, carbon nanotube, ion-beam lithography, new polymer material, electron beam and so on. The records of the biggest cluster 8, carbon nanotube, and 13, scanning tunneling microscopy, are 23 and 22, respectively. 


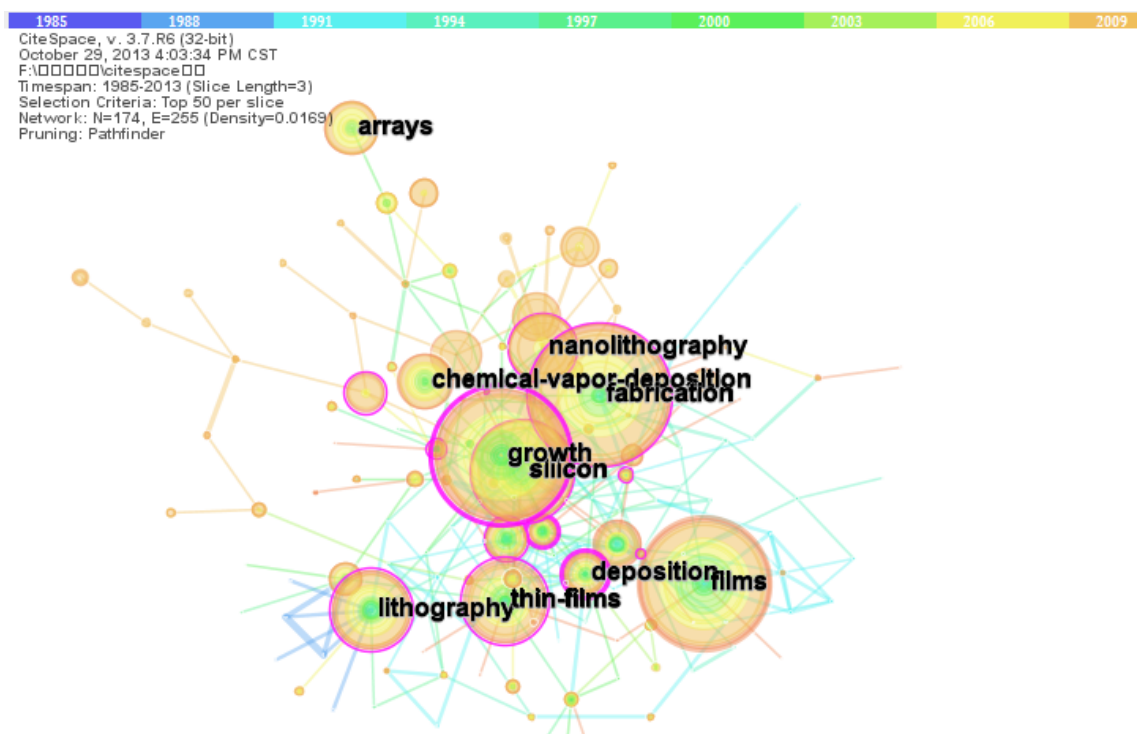

Figure 6. The analysis for hotspots.

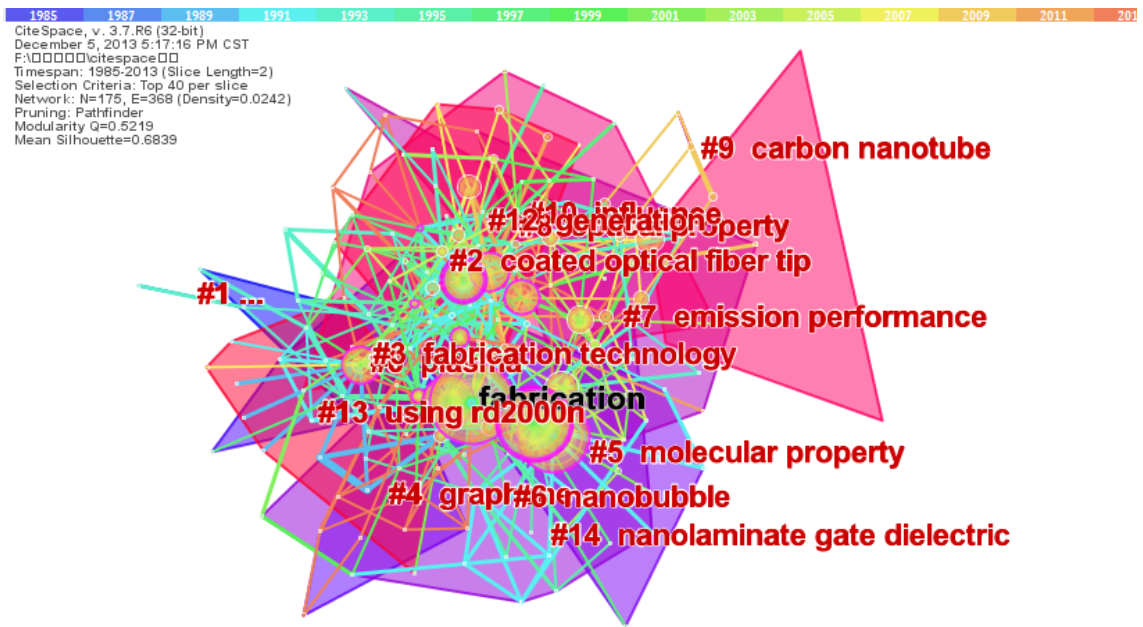

Figure 7. Clusters of the research hotspots.

Table 4. The top 10 table of co-cited keywords.

\begin{tabular}{cccc}
\hline Co-citations & Centrality & Year & Keywords \\
\hline 388 & 0.11 & 1991 & fabrication \\
356 & 0.21 & 1991 & growth \\
354 & 0.07 & 1991 & films \\
293 & 0.12 & 1991 & silicon \\
253 & 0.19 & 1991 & thin-films \\
241 & 0.12 & 1990 & lithography \\
206 & 0.15 & 1991 & nanolithography \\
169 & 0 & 1997 & chemical-vapor-deposition \\
166 & 0 & 2000 & arrays \\
163 & 0.22 & 1991 & deposition \\
\hline
\end{tabular}




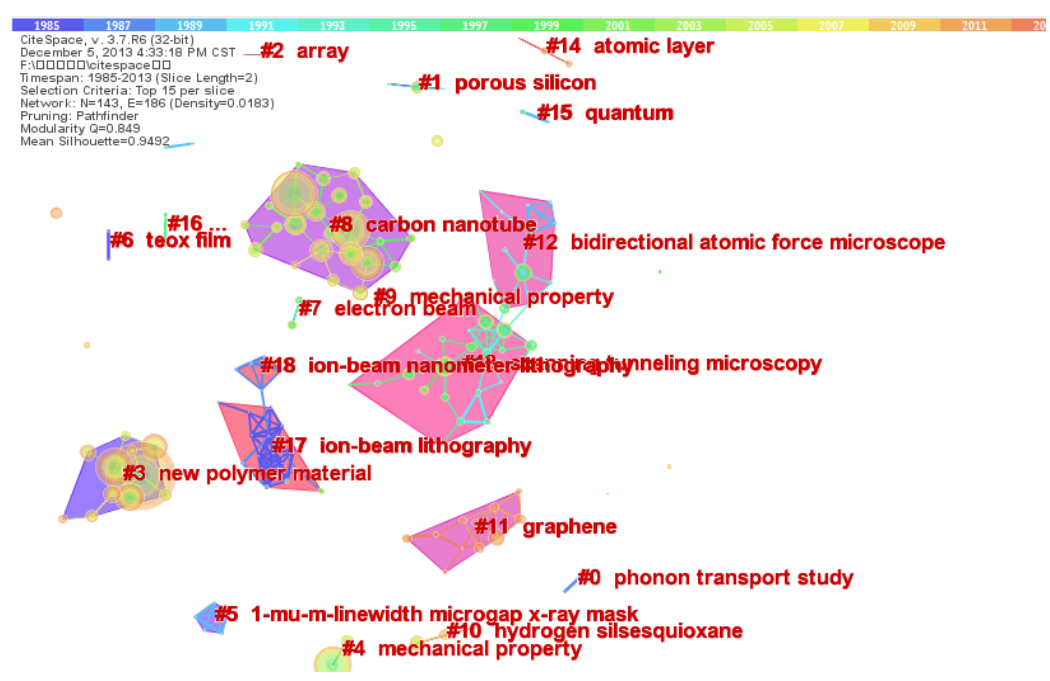

Figure 8. Research fronts clusters.

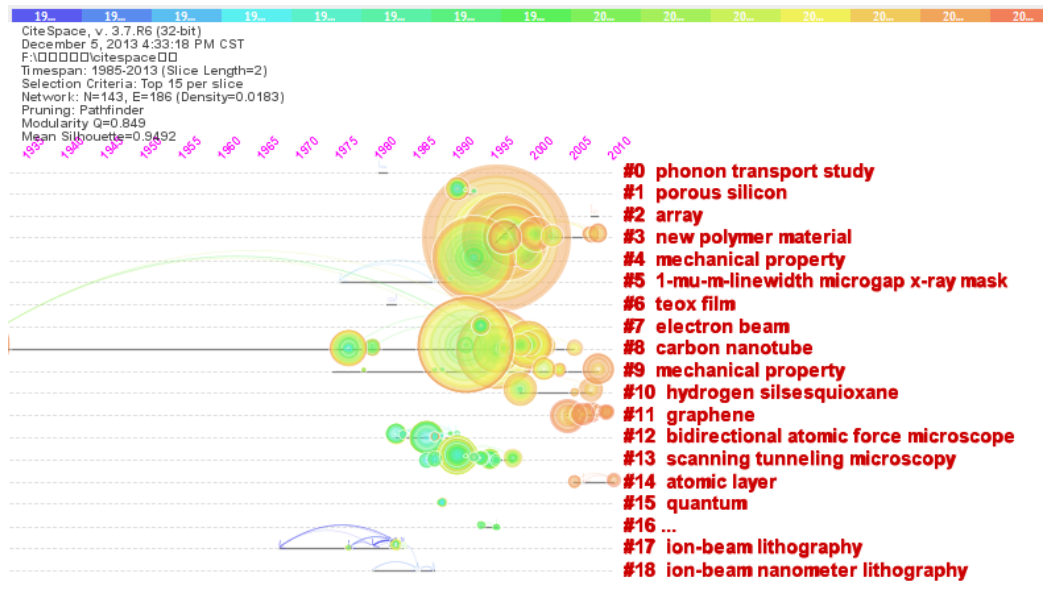

Figure 9. A time-zone view of mass-extinction research.

Table 5. Clusters of the research hotspots.

\begin{tabular}{cc}
\hline Node & Clusters \\
\hline$\# 0$ & plasma \\
$\# 1$ & scanning probe microscope instrument \\
$\# 2$ & coated optical fiber tip \\
$\# 3$ & fabrication technology \\
$\# 4$ & graphene \\
$\# 5$ & molecular property \\
$\# 6$ & nanobubble \\
$\# 7$ & emission performance \\
$\# 8$ & optical property \\
$\# 9$ & carbon nanotube \\
$\# 10$ & influence \\
$\# 11$ & membrane \\
$\# 12$ & generation \\
$\# 13$ & electron-beam nanolithography \\
$\# 14$ & nanolaminate gate dielectric \\
\hline
\end{tabular}


It demonstrates the evolutive gradient path of subject knowledge portal by clicking on the button, "Link Walkthrough", and the color changes of circular node clusters in the map, we get Figure 9. The main referred research before 1990 are ion-beam lithography, ion-beam nanometer lithography, electron beam, carbon nanotube and piezo, and bidirectional atomic force microscope, scanning tunneling microscopy, phonon transport study, porous silicon, mechanical property, microgap X-ray mask, teox film, electron beam and piezo during 1990-2000, and array, new polymer material, carbon nanotube, grapheme, atomic layer between 2000-2010.

\section{Conclusion}

We summarize the nanotechnology references in JVST, and find the institutions ranking top 10 are MIT, IBM CORP, OSAKA UNIV, UNIV ILLINOIS, USN, UNIV WISCONSIN, and CHINESE ACAD SCI. The most influencing author is CHOU SY due to he has a half of 8 core node articles. The research hotspots are fabrication technology, graphene, molecular property, emission performance, electron-beam nanolithography, nanolaminate gate dielectric et al. The research frontals are scanning tunneling microscopy, bidirectional atomic force microscope, carbon nanotube, ion-beam lithography and so on.

\section{References}

[1] Chen, C.M. (2006) CiteSpace II: Detecting and Visualizing Emerging Trends and Transient Patterns in Scientific Literature. Journal of the American Society for Information Science and Technology, 57, 359-377. http://dx.doi.org/10.1002/asi.20317

[2] Hou, H.Y., Liu, Z.Y., Chen, Y., Jiang, C.L., Yin, L.C. and Pang, J. (2006) Mapping of Science Studies: The Trend of Research Fronts. Science Research Management, 27, 90-96.

[3] Zhou, J.X. (2011) Documents Visibilization Analysis of Information Visibilization Based on the Citespace II. Information Sciences, 29, 98-101.

[4] Gong, H. and Lu, Y. (2012) Analysis on the Reseach Evolution Paths of Subject Knowlegy Portals Based on CiteSpace. Journal of Modern Information, 32, 92-96.

[5] Xiao, M., Chen, J.Y. and Li, G.J. (2011) Visualization Analysis on the Research of Mapping Knowledge Domains Based on CiteSpace. Library and Information Service, 55, 91-95.

[6] Hou, H.Y., Liu, Z.Y. and Luan, C.J. (2009) Quantitative Analysis on the Research Front of International Scientometrics Based on Mapping of Knowledge. Science Research Management, 30, 164-170. 